\title{
Desarrollo silvícola de Hidalgo y Puebla en el contexto del Programa Estratégico Forestal para México 2025
}

Matías Edilberto Hernández San Román†

Sergio Gabriel Ceballos Pérez Catedrático Conacyt - El Colegio del Estado de Hidalgo

\section{INTRODUCCIÓN}

El territorio mexicano se caracteriza por su gran riqueza natural, se encuentra dentro del grupo de los 12 países más megadiversos. En el norte y parte del centro del país se localizan las zonas áridas y semiáridas, donde se ubican los matorrales xerófilos, pastizales y bosques espinosos; en las planicies costeras y secas del Pacífico, centro del golfo de México y noroeste de Yucatán se encuentran los bosques tropicales secos y semisecos; en las zonas más húmedas inferiores a los 900 metros sobre el nivel del mar se ubican los bosques tropicales perennifolios, y a mayores altitudes los bosques de niebla; en las sierras se encuentran los bosques de encinos y coníferas (Sarukhan, 2012).

Según datos de la Organización Internacional para la Agricultura y la Alimentación (FAO) 60\% de la superficie forestal mundial se encuentra distribuida entre siete países. México ocupa el octavo lugar a nivel mundial por su extensión forestal que llega a los 55 millones de ha, se encuentra en el segundo lugar entre los países de América Latina, siendo superado en mucho por Brasil que posee una extensión forestal que rebasa los 540 millones de hectáreas [1] JOSÉ, SARUKHÁN, et al. Compilador. Capital natural de México: Acciones estratégicas para su valoración, preservación y recuperación. México, Comisión Nacional para el Conocimiento y Uso de la Biodiversidad, 2012 (De la Mora, 2003).
El 55\% de los bosques se encuentran en manos de comunidades y ejidos de acuerdo con el Registro Agrario Nacional (RAN) (Registro Agrario Nacional 2012). Los estados de Durango, Michoacán, Chihuahua, México, Oaxaca, Puebla, Jalisco, Chiapas, Guerrero y Quintana Roo concentraron $75 \%$ de 1 os permisos de extracción de madera emitidos durante el periodo 1992-2002. De las comunidades con permisos, una décima parte cuentan con capacidad de aserrío y otro tipo de procesamiento industrial (David, 2005), lo que habla de la baja capacidad instalada y el escaso o nulo valor agregado de la madera en beneficio de los propios productores ejidales y comunales (Ceballos, 2010).

\section{Comercio del sector forestal en México}

A partir del Tratado de Libre Comercio que firmo México con Estados Unidos y Canadá, la magnitud del déficit comercial forestal en el país y su tendencia creciente, demuestran la crisis en la que se ha sumido el sector. A partir de la apertura económica, se implementó una política que fue relegando al sector productivo forestal para privilegiar la protección restrictiva de los bosques, la brecha entre consumo y producción maderable creció para el 2000 a 1.84 millones de m3r (28\% con respecto a la producción) (De la Mora, 2003). Esta brecha ha seguido creciendo con el tiempo debido al crecimiento continuado del consumo y a la caída de la producción nacional durante los siguientes años hasta alcanzar 7.24 millones de m3r que representaron $171 \%$ con respecto a la producción para el año 2013. 


\section{Desarrollo silvícola de Hidalgo y Puebla en el contexto del Programa Estratégico Forestal para México 2025}

Detrás de la crisis del sector forestal mexicano y el debilitamiento o desaparición de las empresas, sobre todo las comunitarias, están los impactos que deja en las economías locales, la destrucción de empleos rurales, la pérdida de inversiones por parte de los propietarios para mantener los bosques y selvas protegidos de talamontes, incendios y plagas, y la disminución en los medios de vida de casi 11.5 millones de mexicanos y mexicanas que viven en las regiones forestales. En este contexto de la crisis forestal, el actual gobierno ha implementado la estrategia nacional de producción y productividad forestal. México es deficitario en su producción forestal, ante esta situación dentro del programa estratégico forestal 2025 se ha propuesto como objetivo "... revitalizar la economía del sector forestal, ..."[1]. (Programa Estratégico Forestal 2025, 2016).

\section{EI desarrollo forestal en los estados de Hidalgo y Puebla}

Con el propósito de tener elementos de comparación en torno al desarrollo forestal de los estados de Hidalgo y Puebla se realizó una revisión de las estadísticas que reportan los Anuarios estadísticos de la producción forestal 2000-2012 de la Secretaria de Medio Ambiente y Recursos Naturales (Semarnat, 2013).

En el estado de Hidalgo existe una superficie forestal de 1,072,997 ha de las cuales 230,743 corresponden a una superficie arbolada de bosques de clima frío templado (Figura 1) donde se aprovechan especies maderables, tales como el pino, oyamel, cedro blanco y encino perteneciendo las primeras al grupo de las confieras y el último al grupo arbóreo de las latifoliadas. De esta superficie arbolada en 2004 sólo se explotaban 28,000 ha

Figura No. 1

Superficie forestal de los estados de Hidalgo y Puebla en 2004

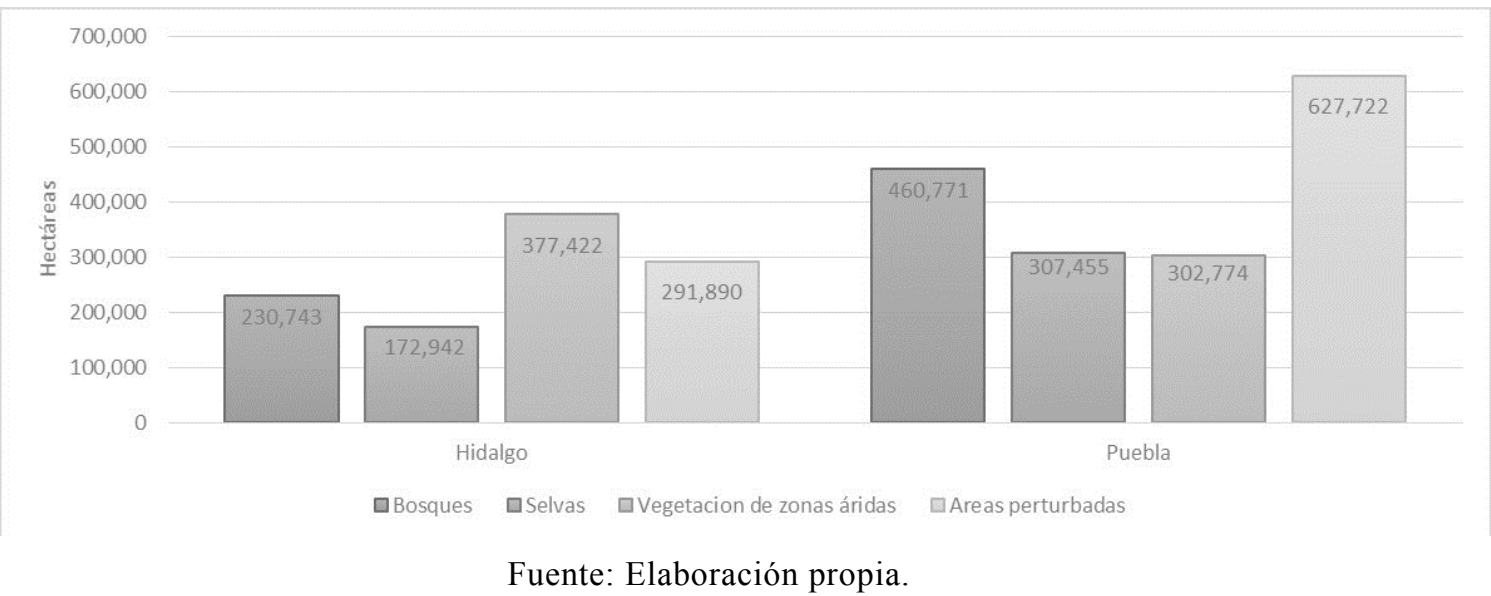




\section{Desarrollo silvícola de Hidalgo y Puebla en el contexto del Programa Estratégico Forestal para México 2025}

que representó el $12 \%$, por lo que existe un potencial del $88 \%$ de dicha superficie que puede incorporarse al aprovechamiento forestal. De esas 28,000 ha el $63 \%$ corresponde a ejidos de la entidad y el restante $27 \%$ a la pequeña propiedad, es decir la mayor parte de los recursos forestales se encuentran ubicados en superficies de propiedad social (Amador, 2003).

La superficie forestal del estado de Puebla comparada con la del estado de Hidalgo es superior por 625,725 ha $(36.84 \%)$, con base a la distribución por tipos de vegetación los bosques y las selvas son mayores en Puebla en un $49.92 \%$ y $43.75 \%$, respectivamente, (Figura 1).

La superficie forestal en el estado de Puebla está conformada principalmente por áreas perturbadas en un $36.95 \%$, seguida de bosques con el $27.12 \%$, selvas con un $18.10 \%$ y con vegetación de zonas áridas en un $17.82 \%$.
Mientras que la superficie de bosques en Hidalgo está conformada principalmente de bosques de latifoliadas, seguido de bosque de coníferas y latifoliadas.

El bosque del estado de Puebla está conformado en su mayoría de bosque de coníferas y latifoliadas. En la Figura 2 se muestra que en los estados de Hidalgo y Puebla las plantaciones forestales no son importantes, pero si el aprovechamiento forestal en los bosques de coníferas y de coníferas y latifoliadas a través de la instrumentación de los programas de manejo forestal.

\section{Autorizaciones de aprovechamiento forestal}

Las autorizaciones de aprovechamiento forestal otorgadas en el estado de Hidalgo descendieron de 1997 al 2000 y aumentaron de 2000 a 2008 como se observa en la Figura 2.

Figura No. 2

Autorizaciones y volumen autorizado de aprovechamiento forestal maderable en el estado de Hidalgo

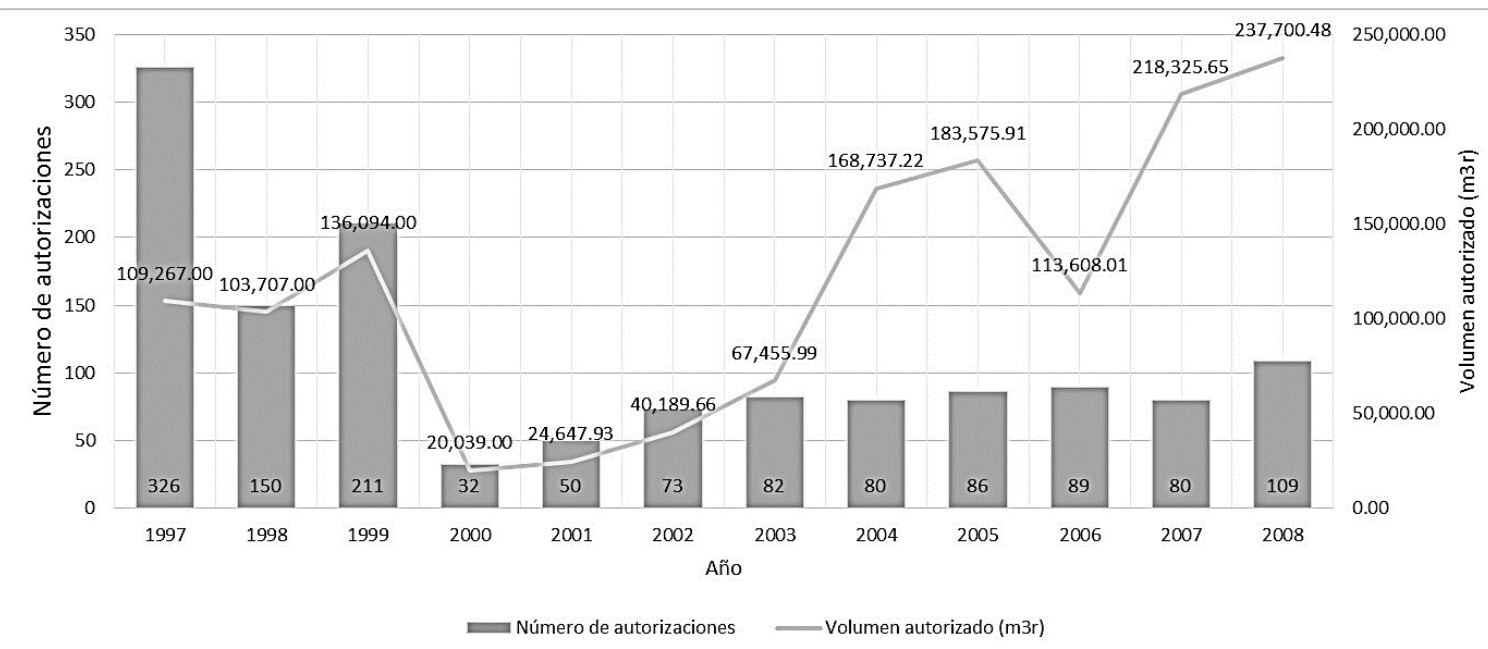

Fuente: Elaboración propia. 


\section{Figura No. 3}

Capacidad instalada y utilizada de la industria forestal maderable en Hidalgo y Puebla en 2000-2004

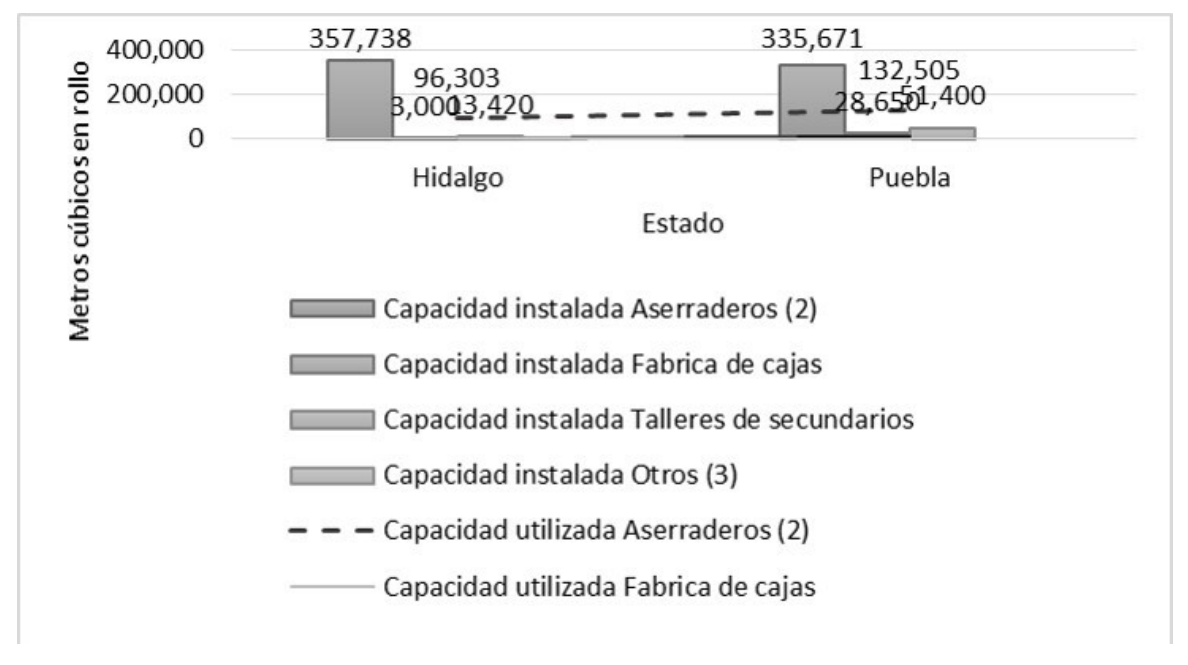

Fuente: Elaboración propia.

En el estado de Puebla la trayectoria del número de autorizaciones ha sido fluctuante a lo largo del periodo de 1997 a 2008, alcanzando el máximo de autorizaciones en 1998 y con un volumen de producción autorizado superior en 1999. Asimismo, se observa que el número de permisos vigentes no es proporcional a los volúmenes de madera que se aprovechan, está en función del tamaño de los predios y de la cantidad de arbolado que marca el inventario se puede extraer.

\section{Industria forestal}

En el estado de Hidalgo existen 1,189 ejidos y comunidades, de los cuales 138 son ejidos con actividad forestal. Desde que entró en operación el programa ProÁrbol, llamado actual PRONAFOR, se han apoyado 31 ejidos y comunidades forestales preferentemente indígenas para la consolidación de proyectos de desarrollo forestal comunitario.
La superficie incorporada al manejo forestal sustentable en ejidos y comunidades del estado de Hidalgo es de 26,429.68 ha (Programa Estatal Forestal 2011-2016., 2012).

La industria forestal en el estado de Hidalgo está conformada en su mayoría por aserraderos en un $90.77 \%$, en menor proporción por fábricas de cajas, talleres secundarios e industrias de la madera. La industria forestal en Puebla está compuesta en su mayoría por aserraderos en un $61.70 \%$, seguida de fábricas de cajas de empaque y talleres secundarios y en menor proporción por fábricas de muebles.

La organización para la producción forestal en Hidalgo y Puebla presenta escaso desarrollo, la mayoría de los productores venden su madera en rollo a pie de brecha. En la (Figura 3) se observa que la capacidad instalada es principalmente de aserraderos en los estados de Puebla e Hidalgo y que la - 


\section{Desarrollo silvícola de Hidalgo y Puebla en el contexto del Programa Estratégico Forestal para México 2025}

industria de cajas, talleres secundarios y otros es incipiente. En relación con la capacidad instalada y utilizada de los aserraderos, se observa que la capacidad utilizada en el estado de Hidalgo es del $26.92 \%$ y en el estado de Puebla del $39.47 \%$, es decir no se ocupa ni el $50 \%$ de la capacidad instalada.

\section{Producción forestal maderable}

En el estado de Hidalgo el volumen de producción maderable de pino de 2008 a 2012 decrecio un $37.42 \%$, mientras que la producción en el estado de Puebla se ha mantenido más o menos constante en el periodo analizado, la producción de Puebla en 2012 fue superior en un $59.10 \%$ respecto a la obtenida en el estado de Hidalgo. En producción de otras especies el estado de Puebla obtiene mayores volumenes, en 2012 obtuvo un $43.49 \%$ más que el estado de Hidalgo, como se observa en la (Figura $4)$.
Ligado a la tendencia de baja en la producción forestal maderable en el estado de Hidalgo, el valor de la producción de madera de pino decrecio en un $40.96 \%$ durante el periodo analizado de 2008 a 2012. El valor de la producción de pino en el estado de Puebla en 2012 fue superior en un $55.99 \%$ a la obtenida en el estado de Hidalgo. Respecto al valor de producción de otras especies, en el estado de Puebla se obtuvo un valor superior en un $61.91 \%$ al que se obtuvo en el estado de Hidalgo en 2012, como se muestra en la (Figura 5).

Durante el periodo 2000-2012 la tendencia de la producción maderable en el estado de Hidalgo ha registrado variaciones, alcanzando su máximo en el año 2008 , de 2000 a 2012 se obtuvo una producción maderable promedio anual de 118,437 m3r. En el estado de Puebla la máxima producción maderable se registro en el año 2000.

Figura No. 4

Autorizaciones y volumen autorizado de aprovechamiento forestal maderable en el estado de Hidalgo

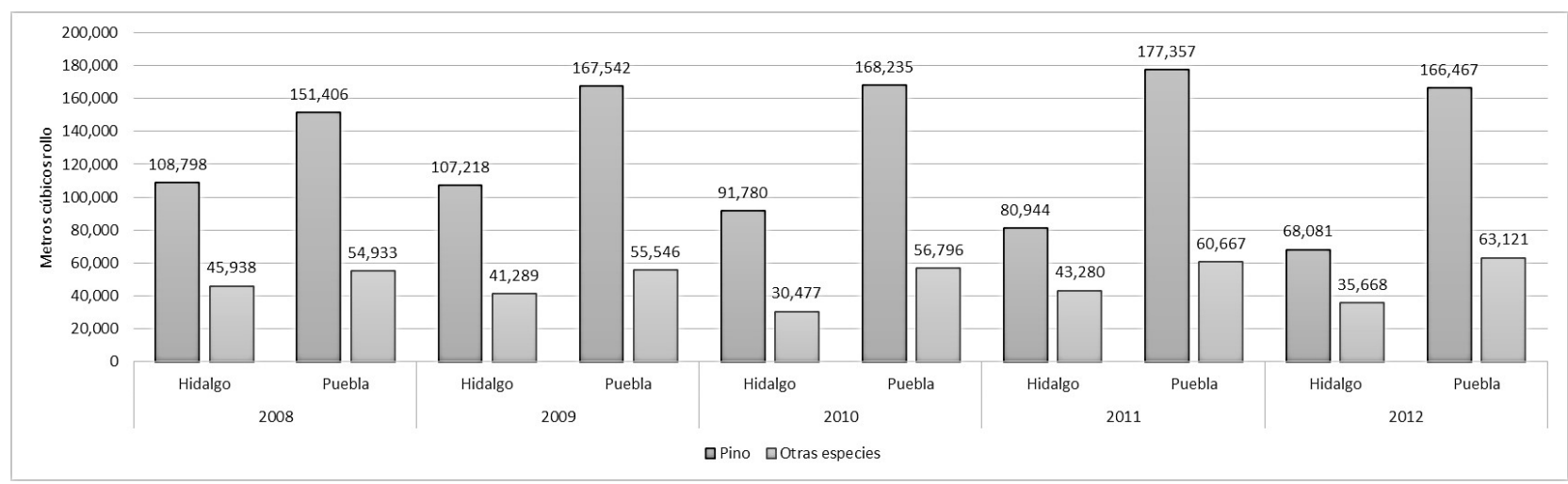

Fuente: Elaboración propia. 
Figura No. 5

Valor en pesos de la producción forestal maderable por género de los estados de Hidalgo y Puebla

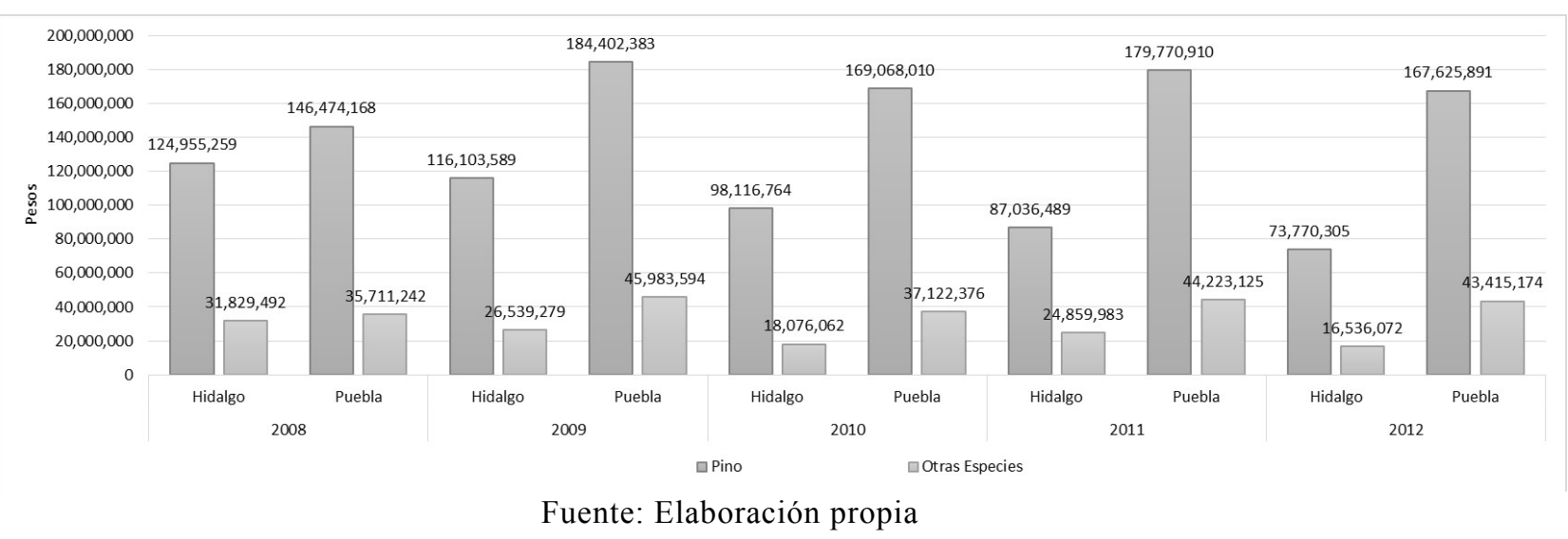

La producción maderable en 2012 en el estado de Puebla fue superior en $125,837 \mathrm{~m} 3 \mathrm{r}$ a la obtenida en el estado de Hidalgo, asimismo el valor de la producción fue superior en el estado de Puebla por $\$ 120,734,688.00$ pesos, respecto al del estado de Hidalgo.

\section{Producción forestal no maderable}

En el estado de Hidalgo la producción forestal no maderable presento su máxima producción en 2011 de otros productos que incluyen hongos, semillas, hojas, nopal, tallos, frutos, musgo, heno, etc. Mientras que el estado de Puebla obtuvo su máxima producción de otras especies no maderables en 2010. Con respecto a la producción forestal no maderable, la producción en el estado de Puebla fue superior en un $25.24 \%$, en relación a la obtenida en el estado de Hidalgo. A pesar de que el volumen de producción es superior en el estado de Puebla, el valor de la - producción forestal no maderable es mayor en el estado de Hidalgo por $\$ 2,539,315.00$ pesos. El valor de la producción forestal no maderable esta compuesto por ingresos obtenidos por la venta de otras especies como son hongos, semillas, hojas, nopal, tallos, frutos, musgo, heno y por la venta de tierra.

\section{Producción maderable por producto}

El principal producto maderable de pino es la escuadría en los estados de Hidalgo yPuebla, en 2012 la producción de escuadría de pino es superior en el estado de Puebla por 83,207 m3r en relación a la producción de Hidalgo. En cuanto al valor de la producción de escuadría de pino tambien es mayor en el estado de Puebla por $\$ 89,661,155.00$ pesos con respecto al del estado de Hidalgo. 


\section{Desarrollo silvícola de Hidalgo y Puebla en el contexto del Programa Estratégico Forestal para México 2025}

En 2012 los principales productos de oyamel en el estado de Puebla e Hidalgo fueron la escuadría y la leña, el estado de Puebla tuvo una producción superior de escuadría de oyamel de $88.52 \%$ en comparación con el estado de Hidalgo, asimismo el valor de la producción obtenido en el estado de Puebla es mayor en un $91.85 \%$ en comparación con Hidalgo.

El estado de Hidalgo tuvo en 2008 y 2012 una mayor producción de escuadría de otras coníferas con un $69.64 \%$ y $87.81 \%$ respectivamente, en comparación con el estado de Puebla, en consecuencia el valor de la producción por la venta de escuadría en el estado de Hidalgo también fue superior con relación a Puebla (Figura 6).

Los principales productos de encino en los estados de Hidalgo y Puebla son la escuadría, la leña y el carbón, siendo la escuadría la que mayores ingresos económicos genera, seguida por el carbón y la leña.
La mayor producción de escuadría de encino se obtuvo en 2011 en el estado de Hidalgo, con una producción superior a la del estado de Puebla. En lo que se refiere a la leña el estado de Puebla tiene una producción relativamente mayor de $9.07 \%$ que Hidalgo y en carbón el estado de Hidalgo tiene una mayor producción en comparación con Puebla; en lo que respecta al valor total de la producción por la venta de encino Hidalgo tiene un valor superior en un $42.8 \%$, por encima de Puebla, esto en 2012.

En ambos estados el principal producto de otras latifoliadas es la leña, seguida de la escuadría. El estado de Hidalgo tiene una producción incipiente en escuadría y leña de otras latifoliadas, mientras que Puebla tiene una producción superior del $89.31 \%$ en escuadría y del $84.18 \%$ en la producción de leña.

Figura No. 6

Valor y producción maderable de otras coníferas por producto en el estado de Puebla e Hidalgo (metros cúbicos rollo) Otros: Celulósicos, carbón, leña y postes, pilotes y morillos

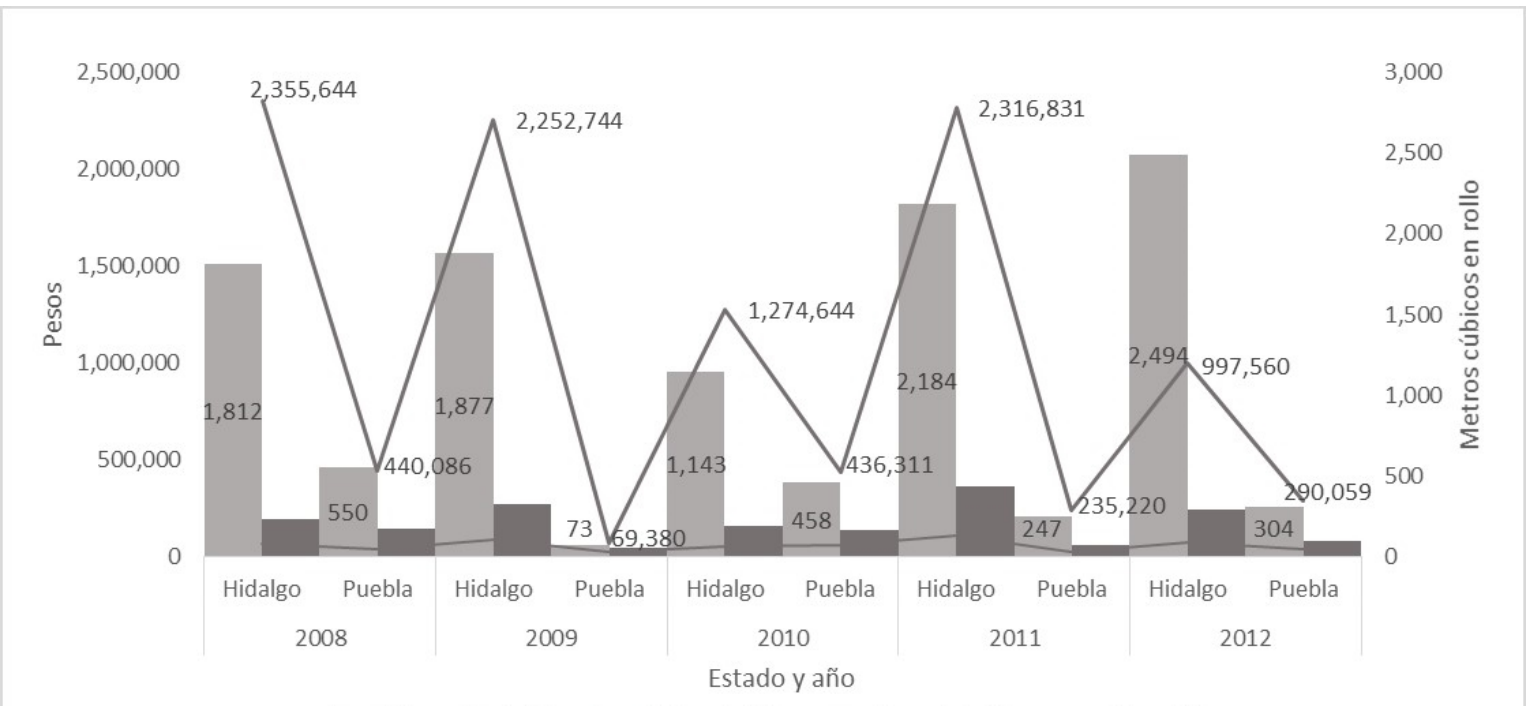

Fuente: Elaboración propia. 


\section{Desarrollo silvícola de Hidalgo y Puebla en el contexto del Programa Estratégico Forestal para México 2025}

Los principales productos en Puebla de maderas preciosas son la escuadría y la leña, con una producción máxima de escuadría y de leña en 2009. El valor máximo de producción se obtuvo en 2009 por la venta de productos de madera preciosa, en el estado de Hidalgo no hubo producción de maderas preciosas en el periodo analizado.

\section{Conclusiones}

Los resultados obtenidos del análisis comparativo en torno a la producción forestal entre los estados de Hidalgo y Puebla muestran la heterogeneidad que presenta el país en torno a la distribución, existencia y las condiciones en que se aprovechan los recursos naturales que poseen las diferentes regiones y estados que integran el territorio nacional. Sin embargo, a pesar de esas heterogeneidades, se aprecian tendencias en torno a la política de desarrollo forestal y lo que se alcanza a percibir en este estudio es el dominio de la producción de escuadría y no de otros productos de mayor valor, lo cual indica que la madera aserrada se produce en ambos estados se le está dando valor en otros lugares, fuera de dichos estados.

El clima, la topografía, la pluviometría y el tamaño de la superficie son factores que influyen sobre la composición florística y el volumen de la producción en los estados de Hidalgo y Puebla; en ambos estados sobresale la producción de coníferas. La capacidad de manejo influye de manera importante para obtener o no mayores volúmenes por unidad de superficie y lo que demuestra el análisis realizado es que ambos - estados a pesar de sus diferencias son importantes para la producción forestal, por ello forman parte de la estrategia nacional del actual gobierno de producción y productividad que integra a 12 estados del país, con recursos forestales de clima templado. El estado de Hidalgo en el periodo analizado poseía un mayor número de aserraderos que Puebla, lo cual no es proporcional al volumen aprovechado, dado que el estado de Puebla posee mayor superficie bajo aprovechamiento forestal y mayor volumen producido, esta situación puede ser un indicador de que parte de la madera que se asierra en el estado de Hidalgo proviene de otros estados. La riqueza obtenida de la actividad productiva forestal no sólo está en la producción primaria, sino en la capacidad de darle mayor valor a la producción obtenida, vía la transformación de los productos maderables, en ambos estados la industria forestal esta poco integrada y desarrollada, provocando con ello menores ingresos provenientes de la actividad forestal.

\section{Bibliografía}

Amador Cesar. (2003). La Explotación de los Recursos Forestales en el Estado de Hidalgo, 1980 - 1999. 5 de septiembre 2015, de Investigación en Ciencias Administrativas, Sitio web: $\mathrm{http}: / /$ www.uaeh.edu.mx/campus/icea/r evista/art2_quinto.htm 


\section{Desarrollo silvícola de Hidalgo y Puebla en el contexto del Programa Estratégico Forestal para México 2025}

Bray David. (et al.) (2005). The community forests of Mexico, managing for sustainable landscapes, Texas EUA, University of Texas Press Austin.

Ceballos Sergio. (2010). Manejo Forestal Comunitario, una alternativa de desarrollo sustentable. En Relaciones básicas entre economía y medio ambiente(s/p). Estado de México: UAEM.

Comisión Nacional Forestal. (2004) Estudio Regional Forestal de la Unidad de Manejo Forestal, Teziutlán, Puebla. 10 octubre de 2015 , de ConaforSemarnat-Asociación regional de silvicultores de Teziutlán Sitio web: http://www.conafor.gob.mx:8080/docum entos/docs/9/3087Estudio\%20Regional $\% 20$ Forestal $\% 20$ de $\% 201 \mathrm{a} \% 20$ Unidad $\%$ 20de\%20Manejo\%20Forestal\%200403. pdf

Consejo Civil Mexicano de Silvicultura Social. (2015). Crisis del Sector Forestal Mexicano, nuevos indicadores y evidencias. 10 febrero de 2016 , de C C M S S S i t i o w e b : http://www.ccmss.org.mx/wpcontent/up loads/2014/10/NOTA 38 Crisis del se ctor forestal mexicano nuevos indicad ores $11081 \overline{4}$.pdf

De la Mora Gabriela. (2003). E1 Comercio internacional y el sector forestal en México. 18 agosto 2014, de C C M S S S i t i o w e b : http://www.ccmss.org.mx/biblioteca/25el-comercio-internacional-y-el-sectorforestal-en-mexico-balanza.html

Ramírez, Eduardo. (2010). La propiedad de la tierra, gobernabilidad y funcionamiento de instituciones locales, condición para la gestión de territorios de propiedad social. 28 agosto de 2014.

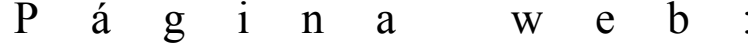
http://media.utp.edu.co/ciebreg/archivos/ taller-internacional-gestion-deterritorios/ramirezppt.pdfSedatu. (2012).

RAN (2012), Propiedad Social, fundamental para el desarrollo de México. 9 mayo de 2012. Página web: http://www.ran.gob.mx/ran/index.php/sa la-de-prensa/historico/1342-propiedadsocial-fundamental-para-el-desarrollode-mexico

Sarukhán José. (2012). Capital natural de México: Acciones estratégicas para su valoración, preservación y recuperación. México, Comisión Nacional para el Conocimiento y Uso de la Biodiversidad.

Semarnat. (2013). Anuarios de producción forestal de 2000 a 2012. 14 septiembre 2014, de Semarnat Sitio web: http://web2.semarnat.gob.mx/temas/gesti onambiental/forestalsuelos/Paginas/anuar ios.aspx

Semarnat. (2014). Programa Estratégico Forestal. 2025, 10 febrero 2016. Sitio w e b : h t t p : / / e r a mx.org/biblio/PEF_2025.pdf

Semarnat. (2016). Autorizaciones de aprovechamiento forestal maderable. 25 septiembre 2015, de Semarnat Sitio web: http://dgeiawf.semarnat.gob.mx:8080/ibi apps/WFServlet?

IBIF ex=D3 RFORESTA03 01\&IBIC _user=dgeia_mce\&IBIC_pass $=$ dgeia_mc e 